\title{
Efficacy of pre-emptive ultrasound guided transverses abdominis plane block with dexamethasone added to bupivacaine for postoperative analgesia after laparoscopic surgeries: A randomised clinical study
}

\author{
Shreyas $\mathbf{S}^{1}$, Shivanand L K ${ }^{2 *}$, Santosh Allalamath ${ }^{3}$, D G Talikoti ${ }^{4}$ \\ ${ }^{1}$ Junior Resident, ${ }^{2}$ Assistant Professor, ${ }^{3}$ Senior Resident, ${ }^{4}$ Professor and HOD, Dept. of Anesthesia, BLDEU Shri B.M. Patil Medical \\ College, Bijapur, Karnataka, India
}

*Corresponding Author: Shivanand L K

Email: shivanandkarigar82@gmail.com

Received: $7^{\text {th }}$ February, 2019

Accepted: $17^{\text {th }}$ March, 2019

\begin{abstract}
Introduction: Laparoscopic surgeries are commonly performed surgical procedures in day to day practice. These patients require a multimodal postoperative pain treatment regimen that provides high quality analgesia with minimal side effects. Opioids, such as morphine, delivered using a patient-controlled analgesia device, remain the mainstay of postoperative analgesic regimens for such patients. TAP block is a regional anaesthetic technique that has been applied as one segment of a multimodal pain regimen in abdominal surgeries. Addition of dexamethasone as adjuvant to bupivacaine in TAP block provides prolonged post-operative analgesia and better pain control than LA alone. The duration of LA was longer with decreased VAS scores and the time of request for rescue morphine doses was less in dexamethasone group.

Objectives: Evaluate efficacy of TAP block in providing post-operative analgesia in laparoscopic surgeries. Evaluate pain severity using VAS. Evaluate need for rescue analgesia. Evaluate safety and adverse effects of TAP block. Group $1-15$ ml $0.25 \%$ Bupivacaine with Dexamethasone $8 \mathrm{mg}$ on both sides if surgical incision involved both sides of rectus sheath. Group $2-15 \mathrm{ml} 0.25 \%$ Bupivacaine with normal saline.

Results: The duration of analgesia increased considerably in study group. The time for request of analgesia was increased (364.7 min) in study group when compared to control group (148.0 min).

Conclusion: dexamethasone to TAP block produces immense post-operative analgesia along with reduced VAS score, increased the duration of analgesia and reduced rescue analgesic dose requirements.
\end{abstract}

Keywords: TAP block, Dexamethasone, VAS score.

\section{Introduction}

Postoperative pain management continues to be a challenge, despite availability of numerous analgesic modalities. Data has shown that $70 \%$ of patients undergoing surgical procedures reported postoperative pain and it is often inadequately treated in the hospital setting. ${ }^{1}$ Untreated or semi-treated postoperative pain is associated with decreased patient satisfaction, delayed patient recovery, longer hospitalizations and increased medical care costs. ${ }^{2}$

Laparoscopic surgeries are commonly performed surgical procedures in day to day practice. These patients require a multimodal postoperative pain treatment regimen that provides high quality analgesia with minimal side effects. Opioids, such as morphine, delivered using a patient-controlled analgesia (PCA) device, remain the mainstay of postoperative analgesic regimens for such patients. However, the use of opioids can result in significant adverse effects including sedation, nausea and vomiting. Multimodal analgesic techniques that use regional anaesthesia and non-opioid pain medications in addition to opioids have shown to decrease opioid consumption as well as its adverse effects. ${ }^{3}$ Some patients are not candidates for regional anaesthesia due to patient refusal, adverse psychological elements, type of surgical procedure, infection at site, pre-existing neurological deficits and bleeding diathesis. Non-opioid pain medications include acetaminophen, aspirin, non-steroidal anti-inflammatory drugs (NSAIDs) and selective cyclo-oxygenase inhibitors. ${ }^{4}$ Use of intravenous NSAIDs, such as ketorolac, has been limited due to side effects including bleeding, gastric mucosal damage and renal toxicity. ${ }^{5,6}$

Transversus abdominis plane (TAP) block is a regional anaesthetic technique that first appeared in the anaesthesia literature in 2001 and has been applied as one segment of a multimodal pain regimen in abdominal surgeries including caesarean sections. TAP block was initially performed as a blind technique, in which a double-pop technique was employed where a blunted needle was passed through the external and internal oblique muscles, a pop was heard as the needle pierced through the fascia overlying the muscle. The needle is inserted in the lumbar triangle of Petit. Main principle of TAP block is to deposit local anaesthetic in plane between internal oblique muscle and transversus abdominis muscle to block the sensory-motor innervations of the anterior abdominal wall which is supplied by anterior rami of the spinal segments T7-T11. A large volume (20 $\mathrm{mL}$ ) of local anaesthetic provides block of the T10 to L1 dermatomes and covers incision for specimen and some port sites. TAP blocks have been used in open and laparoscopic surgery for reductions in pain scores and morphine use (in the first 24 hours), time to tolerating diet, PONV and LOS have been described. ${ }^{7,8}$ Results from recent meta-analysis showed that preoperative TAP blocks provide greater analgesia than postoperative TAP blocks. ${ }^{9}$ 
With the untoward and undesirable side effects of narcotics, regional techniques such as the TAP block offers greater pain relief with less side effects and increased patient satisfaction. The duration of TAP block is limited to the effect of administered local anaesthetics (LAs). However, adjuvants such as epinephrine, ketamine and clonidine are added to LA solution in concentrations advocated for other peripheral blocks to prolong the effect of TAP block with promising results.

Some authors described the addition of dexamethasone which is an efficient glucocorticoid drug with antiinflammatory properties has been proven to prolong effect of local anaesthetics. ${ }^{10}$ In according to several works, ${ }^{11-14}$ the suggested use of ropivacaine as local anaesthetic (prepared as a single $\mathrm{S}$ enantiomer, which provides a longer duration of postoperative analgesia, with a greater margin of safety for cardiotoxicity and neurotoxicity than bupivacaine). Many studies have demonstrated that addition of clonidine to bupivacaine in single-shot TAP block for Caesarean section under SA prolongs analgesia by 10-12 hours and reduces overall postoperative analgesic requirements compared to bupivacaine alone. ${ }^{14,15}$

Further studies have demonstrated that addition of dexmedetomidine to bupivacaine in TAP block provides prolonged post-operative analgesia and better pain control than LA alone. The duration of LA was longer, VAS (visual analogue score) was lower and the need for rescue morphine doses was less when dexmedetomidine was added to bupivacaine. ${ }^{16}$ Many studies have shown that the use of fentanyl as an adjuvant to bupivacaine has significantly reduced intraoperative fentanyl use in terms of both the amount and the number of patients who needed additional doses. ${ }^{17}$ More studies have been done to evaluate the role and potential of dexamethasone and other adjuvants. Glucocorticoids have a perquisite to bind to ligands within the cell and be transported into the nucleus, where they have their effect on DNA transcription. Steroids may potentiate the action of local anaesthetics through modulation of the function of potassium channels in the excitable cells.

The introduction of ultrasound (US) guidance in anaesthesia has permitted an indirect vision of internal structures (muscle, vessels, nerves) and ultrasonography has become an indispensable tool for anaesthesiologist and a gold standard for truncal and peripheral blocks, such as several international guidelines recommend.

Hence this study aims to compare the efficacy of the ultrasound guided Transversus Abdominis Plane (TAP) block by using $0.25 \%$ bupivacaine with dexamethasone versus $0.25 \%$ bupivacaine with normal saline.

\section{Aims and Objectives}

Aims

To observe and compare the analgesic effect of ultrasound guided TAP (transverses abdominis plane) block using $0.25 \%$ bupivacaine with dexamethasone versus $0.25 \%$ bupivacaine with normal saline.

\section{Objectives}

Comparison of effectiveness of ultrasound guided TAP block with $0.25 \%$ Bupivacaine with Dexamethasone and $0.25 \%$ Bupivacaine with normal saline.

\section{Primary Objectives}

1. Evaluate efficacy of TAP block in providing postoperative analgesia in laparoscopic surgeries.

2. Evaluate pain severity using VAS (Visual Analogue Score).

\section{Secondary Objectives}

1. Evaluate need for rescue analgesia.

2. Evaluate safety and adverse effects of TAP block.

\section{Materials and Methods}

This study was conducted in the Department of anaesthesiology, Shri B M Patil Medical College, Hospital and Research Centre, Vijayapura. After obtaining approval of the Institutional Ethical Committee and written informed patient consent 70 adult patients of ASA I and II scheduled to undergo laparoscopic surgeries under general anaesthesia were selected for the study and were administered TAP block.

\section{Study Design}

Prospective Randomized Single Blinded Control Trial.

Method of Collection of Data

Study Design

Prospective randomized clinical trial.

\section{Study Period}

One and half year (December 2016 to June 2018).

\section{Sample Size}

70 patients of both genders randomly divided into two groups of 35 each. (by computer generated random numbers).

\section{Statistical Data \\ Randomisation \\ Done by chit method \\ Study Period}

One and half year from December 2016 to August 2018.

\section{Sample Size}

With anticipated Mean difference of Visual analogue scale as 0.3 between Normal saline and dexamethasone group, anticipated standard deviation as 0.46 the minimum sample per group is 40 with $80 \%$ power and $10 \%$ level of significance

\section{Formula used}

$\mathrm{n}=(\mathrm{Z} \alpha+\mathrm{Z} \beta)^{2} \times 2 \times \mathrm{SD}^{2}$

$\mathrm{Md}^{2}$

$\mathrm{Z}=\mathrm{Z}$ statistics at a level of significance.

$\mathrm{Md}=$ anticipated mean difference. $\mathrm{SD}=$ anticipated standard devience.

Statistical tests used -Chi squire test, ANOVA test, Mean+- SD

\section{Inclusion Criteria}

Patients between 18-60 years of age, ASA $1 \& 2$, either male or female, $\mathrm{BMI}<30 \mathrm{~kg} / \mathrm{m} 2$. 


\section{Exclusion Criteria}

Patient refusal, H/o allergy and contraindication to studied medication or anaesthetic agents, Infection at local site, Patient's inability to describe postoperative pain to investigator (dementia, delirium, psychiatric and neurological disorder, BMI > 30, Pregnancy.

Patients between age group of 18-60 years were randomly allocated into two groups of 35 patients each. Patients were randomized by sealed envelopes to undergo TAP block. Group 1 \& 2 patients both underwent laparoscopic surgeries under general anaesthesia. Preoperative anaesthesia assessment was made after hematological, biochemical tests along with ECG and chest $\mathrm{X}$-ray and general physical examination. At the preoperative visit on the evening of or before surgery, the VAS scale scoring system was explained to all patients. Written informed consent was obtained from all patients to be included in the study. Pre-medication was given as Inj. Midazolam 0.02-0.03 mg/kg IV in the operation theatre. All the patients received a standardized general anaesthesia as per institute protocol. Anaesthesia was induced with propofol $2-4 \mathrm{mg} / \mathrm{kg}$, fentanyl $2 \mathrm{mcg} / \mathrm{kg}$ and atracurium 0.5 $\mathrm{mg} / \mathrm{kg}$ intravenous (IV) and anaesthesia was maintained with isoflurane and $40 \%$ oxygen in nitrous oxide. The study drug was administered according to the group allocated. Patients were reversed with neostigmine and glycopyrollate. Standard monitoring including non-invasive blood pressure monitoring, arterial oxygen saturation, electrocardiogram and end tidal carbon dioxide monitoring were done.

Group 1: TAP block (after induction of General Anaesthesia) with $15 \quad \mathrm{ml} \quad 0.25 \%$ Bupivacaine and Dexamethasone $8 \mathrm{mg}$.

Group 2: TAP block (after induction of General Anaesthesia) by $15 \mathrm{ml} 0.25 \%$ Bupivacaine in $5 \mathrm{ml}$ normal saline.

Patients of both groups were followed during the postoperative period for pain and any adverse events. Pain was assessed by Visual analogue scale $(0-10)$. If patient experienced pain they were asked to grade the pain at rest and on movement. The time was tabulated in serial proportions, calculating from the end of the surgery and patients were followed for 24 hours and the results of 2 groups were compared. For TAP block the skin was prepared with $10 \%$ Betadine solution and a high-frequency (5 - $10 \mathrm{MHz}$ ) ultrasound probe (SonoSite M-Turbo. SonoSite, Inc, Bothwell, MO, USA). The probe was placed horizontally across the abdomen. The muscle layers in the antero-lateral part of the abdomen was traced by scanning from the midline towards the area between the iliac crest and the costal margin in the mid-axillary line.

The rectus abdominis muscle was identified, just off the midline, as an oval / elliptical structure. The ultrasound transducer was moved to scan laterally where the 3 muscle layers could be seen running parallel to one another. With an adequate ultrasound image, the regional block needle was inserted anterior to the transducer. The needle was placed in the Transversus abdominis plane and $2 \mathrm{ml}$ of $0.9 \%$ normal saline was given and splitting of plane was confirmed before injecting $15 \mathrm{ml}$ of $0.25 \%$ bupivacaine (with Dexamethasone or normal saline) slowly. After injection, the fascial plane was seen to separate and from a well-defined hypoechoic elliptical shape between the internal oblique and transversus abdominis muscles.

Patient's baseline vital parameter heart rate, blood pressure, oxygen saturation were noted during surgery, every fifteen minutes for the first hour and later third hourly after surgery, for 24 hours. The findings were noted by a blinded observer. After completion of the surgical procedure and emergence from anaesthesia, patients were transferred to the postoperative recovery room for further monitoring of post-operative pain.

In postoperative recovery room, all patients were monitored for heart rate, blood pressure, oxygen saturation, pain (VAS) and PONV. Rescue analgesia was given Inj. Paracetamol (1gm) intravenously every $8 \mathrm{hrs}$ and Inj. Pentazocine $(30 \mathrm{mg}$ ) intramuscularly in case of visual analogue scale $(\mathrm{VAS}) \geq 4$. Time at which patient demanded rescue analgesia was noted and the VAS score at that time was also noted. Pain was assessed by visual analogue scale (VAS).

\section{Visual Analogue Scale}

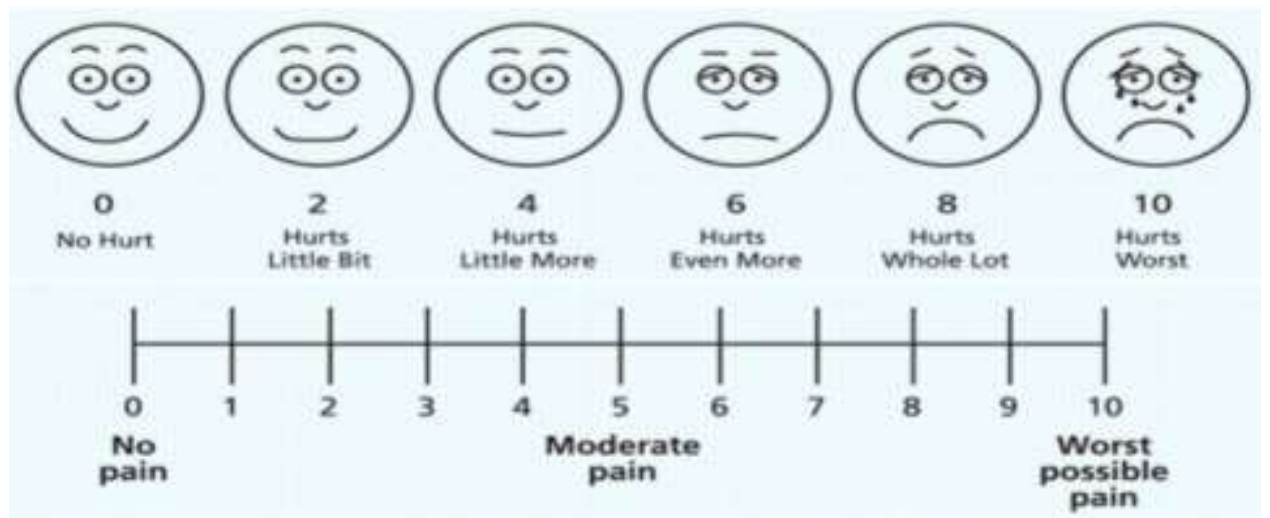

PONV Score

$0=$ no nausea/vomiting, $1=$ nausea/retching, $2=$ Vomiting, $3=$ severe vomiting $/$ projectile . 


\section{Statistical Analysis}

The statistical analysis was done using SPSS Windows version 16.0 software. For non-continuous data Chi-square test was used. The mean and standard deviation of the parameters studied during observation period were calculated for two treatment groups and compared using student ' $\mathrm{t}$ ' test. The critical value of ' $\mathrm{p}$ ' indicating the probability of significant difference was taken as $<0.05$ for comparisons. If the p-value was $<0.05$, then the results were considered to be statistically significant otherwise it was considered as not statistically significant.

\section{Results}

Our study included a total of 70 patients of both gender randomly divided into two groups of 35 each. Patients were assigned to two groups randomly with computer allotted numbers. Out of 70 patients, 36 were males and 34 patients were female distributed in both study group and control groups. (Table 1)

Total of 70 ASA class I and II subjects were studied. In the study group 28 patients were belonging to ASA class I and rest were of class II, where as in the control group 31 patients belonged to ASA class I and 4 were in class II. (Table 2) Our study showed that, TAP block with dexamethasone or normal saline is not influenced by ASA class of the patient undergoing the Block/procedure.

We assessed parameters like Age/Weight/Height/BMI for influencing the duration of post op analgesia with TAP block in both groups. Statistical significance was not noted indicating that these parameters did not influence duration of post op analgesia with TAP block in both the groups. (Table 3)

The mean of total duration of surgery was 89.6 minutes in study group and 84.1 minutes in the control group. This duration of surgery was assessed because it influenced the duration of post op analgesia with TAP block in both groups. No statistical $\mathrm{P}$ value determined in our study. (Table 4)

Heart rate was assessed among all subjects for influencing the duration of post op analgesia with TAP block in both groups. Mean heart rate was considered before surgery, during surgery, immediate postop and at intervals1hour, 2 hour, 4hours, 8 hours, 12 hours and 24 hours postoperatively in both the groups with no statistical significance noted.

Systolic blood pressure (Table 5) and Diastolic blood pressure (Table 6) was assessed for influencing the duration of post op analgesia with TAP block in both groups. Mean SBP and DBP were taken before surgery, during surgery, immediate postop and at intervals of -1 hour, 2 hour, 4 hours, 8 hours, 12 hours and 24 hours postoperatively in both the groups. No statistical significance noted between both the groups.

Standard oxygen saturation parameters were assessed for influencing the duration of post op analgesia with TAP block in both groups. Statically there was no significant difference between the groups. (Fig. 1)
On calculation of mean VAS score in both comparative groups statistical significance noted on $4^{\text {th }}$ and $12^{\text {th }}$ hour post-operative period in a group with dexamethasone as adjuvant with TAP block, indicating increased duration of post-op analgesia in dexamethasone group. (Fig. 2)

Total duration of analgesia and time request of analgesia was assessed with TAP block in both groups. The duration of analgesia increased considerably in study group. The time for request of analgesia was increased $(364.7 \mathrm{~min})$ in study group when compared to control group (148.0 min). (Fig. 3)

On calculation of mean VAS at T rescue score and after comparison between both the groups statistical significance noted in post-operative period in the group with dexamethasone as adjuvant in TAP block, indicating increased duration of post-op analgesia (Fig. 4)

Statistical significance was noted in the $4^{\text {th }}$ hour for both groups and the need for post-operative analgesia was compared where in seven subjects needed analgesics (20\%) in dexamethasone group compared to twenty -nine subjects required analgesics $(82 \%)$ in normal saline group. Similar difference noted in $6^{\text {th }}$ and $12^{\text {th }}$ hour. Hence TAP with adjuvant dexamethasone is efficacious and superior in postoperative analgesic management. (Fig. 5)

On calculation of PONV score in both comparative groups statistical significance noted during $1^{\text {st }} / 2^{\text {nd }} / 4^{\text {th }}$ and $6^{\text {th }}$ hour post-operative period, with highest incidence in $2^{\text {nd }}$ hour with four patients experiencing PONV in the group with normal saline as adjuvant to TAP block, indicating less incidence of post-op nausea and vomiting in dexamethasone group. (Fig. 6)

Table 1: Distribution of cases according to sex between study groups

\begin{tabular}{|c|c|c|c|c|c|}
\hline \multirow{2}{*}{ Sex } & \multicolumn{2}{|c|}{$\begin{array}{c}\text { Study } \\
(\mathbf{N}=35)\end{array}$} & \multicolumn{2}{|c|}{ Control $(\mathbf{N}=35)$} & \multirow{2}{*}{ p value } \\
\cline { 2 - 5 } & $\mathbf{N}$ & $\mathbf{\%}$ & $\mathbf{N}$ & $\mathbf{\%}$ & \\
\hline Male & 22 & 62.9 & 14 & 40.0 & \multirow{2}{*}{0.056} \\
\hline Female & 13 & 37.1 & 21 & 60.0 & \\
\hline Total & 35 & 100.0 & 35 & 100.0 & \\
\hline
\end{tabular}

Table 2: Distribution of cases according to ASA class between study groups

\begin{tabular}{|c|c|c|c|c|c|}
\hline \multirow{2}{*}{$\begin{array}{c}\text { ASA } \\
\text { Class }\end{array}$} & \multicolumn{2}{|c|}{ Study $(\mathbf{N}=\mathbf{3 5})$} & \multicolumn{2}{|c|}{ Control $(\mathbf{N}=35)$} & \multirow{2}{*}{ p value } \\
\cline { 2 - 5 } & $\mathbf{N}$ & $\mathbf{\%}$ & $\mathbf{N}$ & $\mathbf{\%}$ & \\
\hline I & 28 & 80.0 & 31 & 88.6 & \multirow{2}{*}{0.324} \\
\hline II & 7 & 20.0 & 4 & 11.4 & \\
\hline Total & 35 & 100.0 & 35 & 100.0 & \\
\hline
\end{tabular}


Table 3: Comparision of mean demographic parameters between study groups

\begin{tabular}{|c|c|c|c|c|c|}
\hline \multirow{2}{*}{ Demographic Parameters } & \multicolumn{2}{|c|}{ Study } & \multicolumn{2}{c|}{ Control } & \multirow{2}{*}{ p value } \\
\cline { 2 - 5 } & Mean & SD & Mean & SD & \\
\hline Age (Years) & 32.4 & 12.1 & 32.6 & 11.7 & 0.952 \\
\hline Weight (KG) & 57.0 & 5.7 & 55.1 & 9.6 & 0.322 \\
\hline Height (CM) & 161.3 & 6.6 & 160.5 & 7.7 & 0.68 \\
\hline BMI & 22.0 & 2.7 & 21.4 & 3.2 & 0.416 \\
\hline
\end{tabular}

Table 4: Comparision of mean duration between study groups

\begin{tabular}{|c|c|c|c|c|c|}
\hline \multirow{2}{*}{ Duration } & \multicolumn{2}{|c|}{ Study } & \multicolumn{2}{c|}{ Control } & \multirow{2}{*}{ p value } \\
\cline { 2 - 6 } & Mean & SD & Mean & SD & \\
\hline Total duration surgery & 89.6 & 17.6 & 84.1 & 7.0 & 0.094 \\
\hline
\end{tabular}

Table 5: Comparision of mean SBP according to time

\begin{tabular}{|c|c|c|c|c|c|}
\hline \multirow{2}{*}{ SBP } & \multicolumn{2}{|c|}{ Study } & \multicolumn{2}{c|}{ Control } & \multirow{2}{*}{ p value } \\
\cline { 2 - 5 } & Mean & SD & Mean & SD & \\
\hline Baseline & 123.4 & 9.5 & 120.8 & 8.4 & 0.237 \\
\hline During surgery & 115.3 & 9.2 & 113.0 & 7.3 & 0.254 \\
\hline Immediate postop & 116.9 & 10.0 & 115.8 & 6.6 & 0.592 \\
\hline $1 \mathrm{hr}$ & 116.3 & 7.7 & 116.0 & 6.6 & 0.868 \\
\hline $2 \mathrm{hr}$ & 116.9 & 8.7 & 116.5 & 7.7 & 0.817 \\
\hline $4 \mathrm{hr}$ & 118.2 & 8.1 & 117.4 & 7.5 & 0.648 \\
\hline $6 \mathrm{hr}$ & 120.2 & 8.3 & 117.1 & 7.2 & 0.1 \\
\hline $12 \mathrm{hr}$ & 119.2 & 7.7 & 118.7 & 7.8 & 0.783 \\
\hline $24 \mathrm{hr}$ & 117.9 & 6.2 & 117.7 & 7.3 & 0.888 \\
\hline
\end{tabular}

Table 6: Comparision of mean DBP according to time

\begin{tabular}{|c|c|c|c|c|c|}
\hline \multirow{2}{*}{ DBP } & \multicolumn{2}{|c|}{ Study } & \multicolumn{2}{c|}{ Control } & \multirow{2}{*}{ p value } \\
\cline { 2 - 5 } & Mean & SD & Mean & SD & \\
\hline Baseline & 81.5 & 10.9 & 82.5 & 8.1 & 0.655 \\
\hline During surgery & 75.5 & 8.8 & 74.9 & 8.1 & 0.779 \\
\hline Immediate postop & 73.6 & 7.6 & 74.6 & 7.6 & 0.595 \\
\hline $1 \mathrm{hr}$ & 72.2 & 6.3 & 72.9 & 7.8 & 0.698 \\
\hline $2 \mathrm{hr}$ & 71.7 & 7.0 & 73.9 & 7.3 & 0.186 \\
\hline $4 \mathrm{hr}$ & 71.7 & 7.0 & 74.9 & 6.7 & 0.056 \\
\hline $6 \mathrm{hr}$ & 73.3 & 6.7 & 76.5 & 5.9 & 0.041 \\
\hline $12 \mathrm{hr}$ & 75.5 & 9.0 & 78.3 & 6.3 & 0.136 \\
\hline $24 \mathrm{hr}$ & 76.2 & 9.3 & 77.7 & 7.3 & 0.458 \\
\hline
\end{tabular}

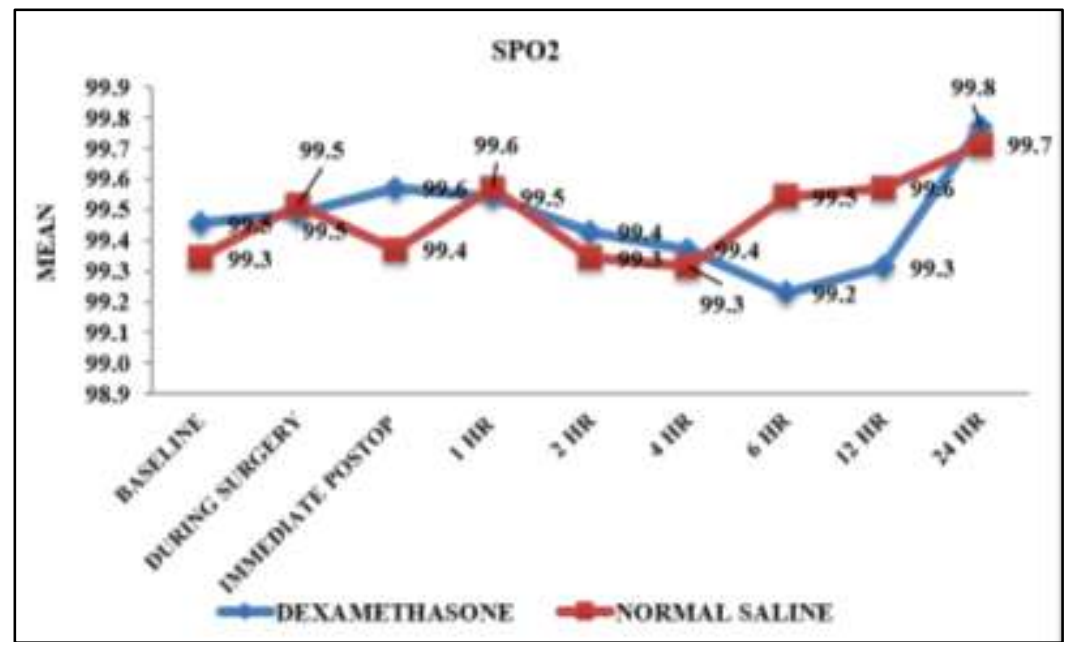

Fig. 1: Comparision of mean Spo2 according to time 


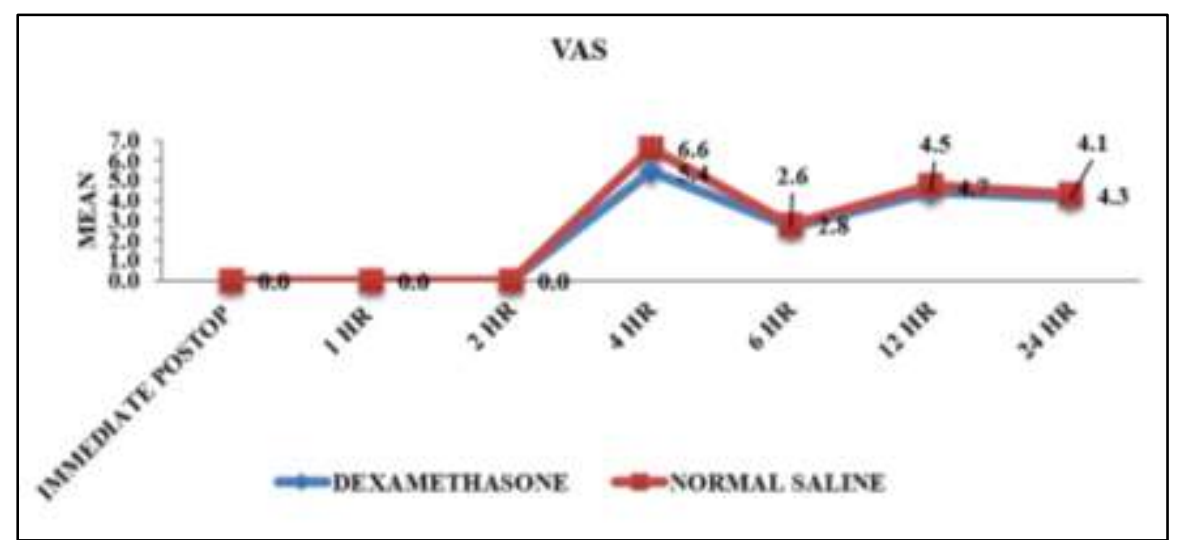

Fig. 2: Comparision of mean vas according to time

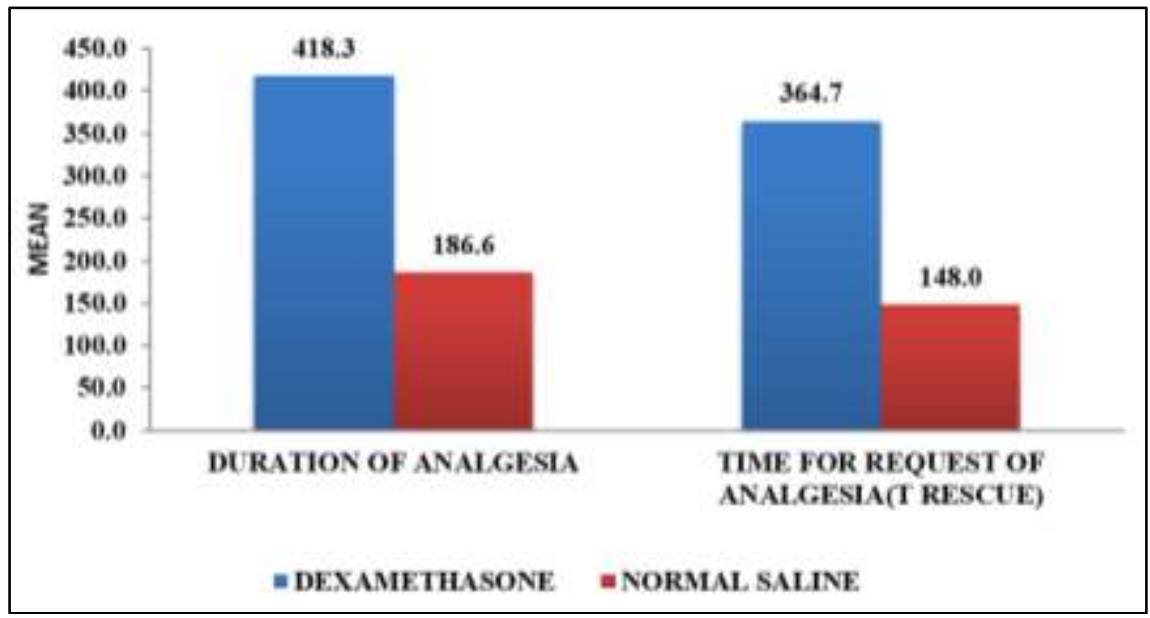

Fig. 3: Comparision of mean parameters according to time

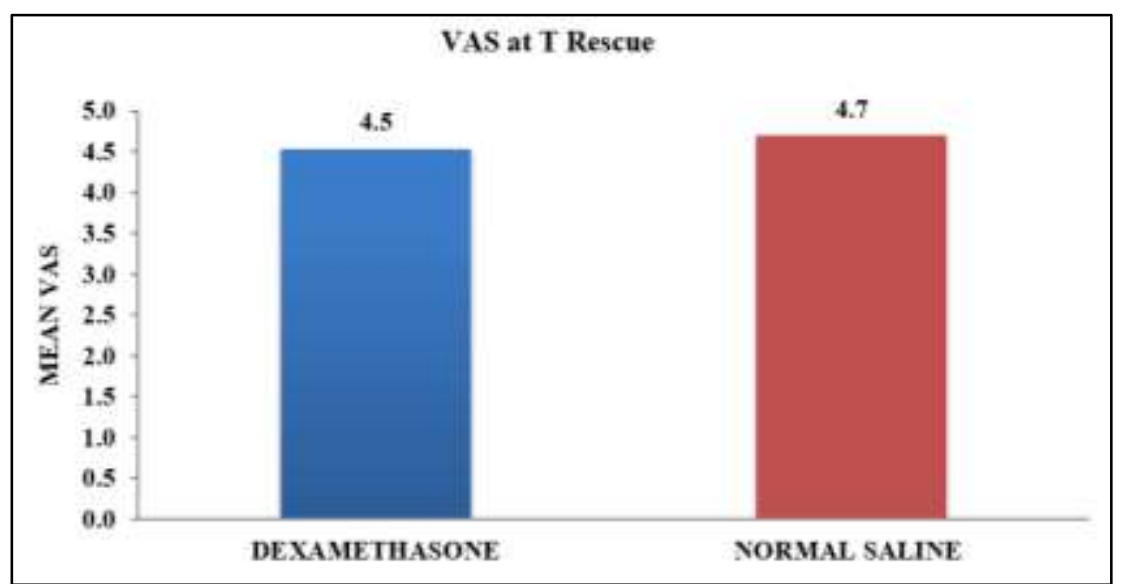

Fig. 4: Comparision of mean vas at $t$ rescue according to time 


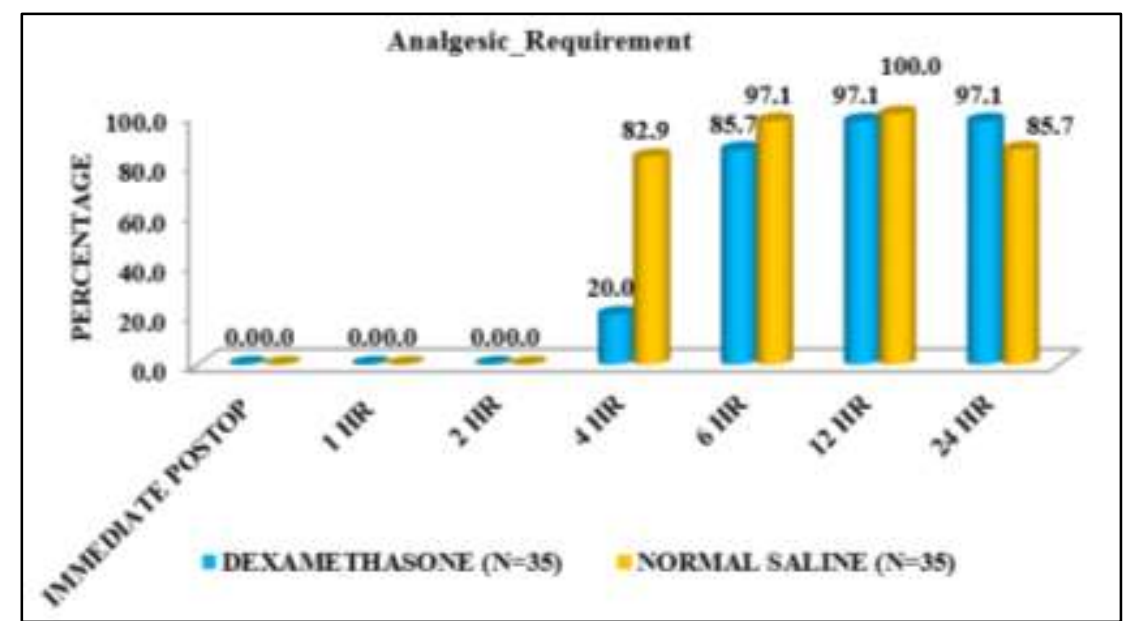

Fig. 5: Analgesic requirement between study groups

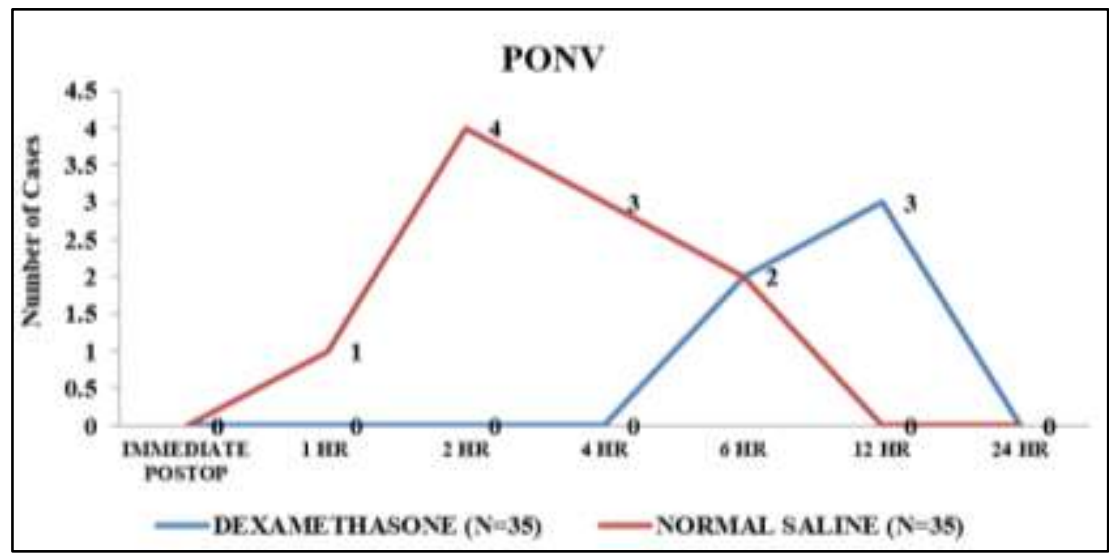

Fig. 6: PONV (> 1) between study groups

\section{Discussion}

This randomized clinical trial demonstrated that the TAP block with dexamethasone as an adjuvant added to bupivacaine when used as a part of multimodal analgesia provides effective analgesia for patients undergoing laparoscopic surgery. It reduced the intensity of break through pain and requirement of opioids postoperatively. All blocks were done under ultrasound guidance which ensured the exact location. There was no block related complication.

The standard regimen of paracetamol, opioid and NSAID at our institution didn't provide good postoperative pain relief in all patients undergoing laparoscopic surgeries. Substantial component of pain experienced by the patient is from abdominal wall incision in abdominal surgeries any interventions that block pain from abdominal wall will provide good post-operative pain relief so a multimodal analgesia regimen is needed for providing effective postoperative pain relief. Adding dexamethasone to the block increases the duration of the block thereby providing increased duration of analgesia and reduced incidence of PONV.

In the postoperative period the intensity of pain was assessed by VAS scoring system. On the first complaint of pain by the patient or a VAS score of more than 4 a rescue analgesic was administered and the time was noted. The time elapsed between the administration of the block and the first rescue analgesic was recorded as the duration of postblock analgesia. The VAS scores were recorded every second hour from the time of drug administration. The VAS scores in the dexamethasone group $(\mathrm{V} 6 \mathrm{~h}=3.70 \pm 0.68)$ were comparable with the VAS scores noted with the studies done by Ra et al. ${ }^{18}(\mathrm{~V} 6 \mathrm{~h}=3.1 \pm 1.55)$ and Aveline et al. ${ }^{19}$ who employed the same concentration in their studies. The intensity of pain as experienced by the patient in the postoperative period was lesser in the dexamethasone group than in the normal saline group. In a qualitative study by Nurçin Gülhaş et al. ${ }^{20}$ in 2015 demonstrated that the addition of dexamethasone to ultrasound guided TAP block had decreased postoperative pain scores, increased the time to first analgesic requirement, reduced postoperative narcotic requirements and adverse events related to it. The incidence of nausea or vomiting was also reduced with shorter length of hospital stay in patients undergoing total abdominal hysterectomy. TAP Block with dexamethasone group had reduced VAS Scores throughout the $24 \mathrm{hr}$ postoperative period. The patients in TAP Group had significantly decreased VAS scores $(p<0.05)$ for $24 \mathrm{hr}$ period except at $0,1 \mathrm{hr}$ postoperative period with a mean VAS Score of 2 . Similarly 
decreased VAS Scores was also observed by Mc Donnell et al. ${ }^{21}$ G. Niraj et al. ${ }^{22}$

The duration of post-block analgesia was defined as the time from the injection of the LA to the time of first complaint of pain by the patient, at which point he/she received Injection Pentazocine $30 \mathrm{mg}$ IM. In the normal saline group this duration was found to be $186.6 \pm 67.6 \mathrm{~min}$ and in dexamethasone group it was found to be $418.3 \pm 94.9$ $\min$. The difference in the duration of analgesia provided by the two groups was found to be statistically significant $(P<$ $0.05)$. In our study, the patients who received the TAP block had a significant reduction in the post-operative rescue analgesics consumption $(p<0.03)$ at 2,4 and 6 hrs. The duration of TAP block is influenced by the efficacy of LA given and the dose administered, also the maximum permitted dose for that agent plays a role in accordance to toxicity of the drug. (rephrase the sentence) This has led to the use of adjuvants such as clonidine, dexmedetomidine to prolong the effect of LA in TAP block. In a randomized control study, $\mathrm{MgSO}_{4}(150 \mathrm{mg}$ ) was added as an adjuvant to bupivacaine in USG-guided TAP block proved that it reduces post-operative pain scores, prolongs the duration of analgesia and decreases demand for rescue analgesics. ${ }^{6}$ In another randomized controlled trial which demonstrated that addition of clonidine to bupivacaine in single-shot TAP block for cesarean section under SA prolongs analgesia by 10-12 hour and reduces overall postoperative analgesic requirements by more than $75 \mathrm{mg}$ compared to bupivacaine alone. ${ }^{7}$ In another study by Almarakbi et al. the addition of dexmedetomidine to bupivacaine in TAP block achieves better local anaesthesia and provides better pain control post-operatively without any major side-effects. ${ }^{23}$ In one study the effects when fentanyl, when added as an adjuvant suggested that the addition of only $2.5 \mu \mathrm{g} / \mathrm{ml}$ fentanyl to the TAP block procedure $(0.375 \%$ ropivacaine $)$ was unable to improve the duration and quality of analgesia after caesarean delivery. In contrary to the point that the cumulative fentanyl consumption was significantly lower in both the groups postoperatively. ${ }^{24}$

The opioids sparing effect has been documented by Niraj et al. ${ }^{22}$ In their study with TAP block which had the similar result of reduced opioids consumption has been observed in our analysis of the data of TAP block in laparoscopic surgery. ${ }^{25}$ Indicating the reduced incidence of the opioids-related side effects.

Time to rescue analgesia was prolonged in our study in all the subcategories of laparoscopic surgeries where TAP block was administered which was in accordance with the study of McDonnell et al. ${ }^{26}$ and Carney et al. ${ }^{27}$

This shows the effectiveness of TAP block as a part of multimodal analgesia regimen and its ability of reducing opioid requirement and opioid related adverse effects. In our study we found that addition of dexamethasone to TAP Block, showed reduced analgesics requirement during 24 hour period in comparison to TAP block with normal saline. The TAP block reduced the pain score with its ability to block transmission of nociceptive impulse from abdominal wall. This shows that adding dexamethasone to TAP Block can increase duration of the block by providing good pain relief for a period of $24 \mathrm{hrs}$. This is in accordance with study by El Sharnouby et al. ${ }^{28}$

TAP block also reduced the incidence of PONV. As for post-operative pain management the use of opioids results in PONV. Pain and postoperative nausea and vomiting top the list of causes of dissatisfaction. Our analysis shows that not only analgesic requirements in TAP block are lower but also the pain scores and the PONV incidence is also significantly smaller. Many direct and indirect factors could have contributed toward this desirable outcome for PONV. Other than direct relation of opioids consumed, higher pain scores are known to directly increase PONV as well. Supporting our study included the analysis by Soltani Mohammadi et al. ${ }^{28}$ and Parikh et al. ${ }^{29}$ demonstrated that overall numeric rating scale (NRS) for pain had significantly lower values with the use of TAP block because it provided analgesia for longer duration. Thereby limiting the use of opioid for postoperative pain ultimately reducing the incidence of PONV. The addition of dexamethasone a potent anti-emetic was also a contributing factor. PONV scale is graded by four points from 0 (being no incidence) to 3 (having recurrent incidence). In calculating the incidence of PONV, any score of above zero at any time point was taken as indicator that the patient had PONV. Many clinical studies also report similar reduction in PONV incidence, McDonnell et al. ${ }^{26}$

Although analgesic requirements in the study (dexamethasone) group increased in comparison to the control (normal saline) group during the 24 hour period. The statistically significant point being less usage of analgesics in the study group which signified a prolonged duration of the block.

\section{Conclusion}

TAP Block is easy to perform under ultrasound guidance without complication and it provides effective analgesia. Adding dexamethasone as an adjuvant to TAP block produces immense post-operative analgesia with concomitant increase in duration of analgesia with reduced VAS score and rescue analgesic dose requirements. It also reduces PONV considerably.

\section{Conflict of Interest: None.}

\section{References}

1. Pergolizzi JV, Raffa RB, Tallarida R, Taylor R, Labhsetwar SA. Continuous multimechanistic postoperative analgesia: a rationale for transitioning from intravenous acetaminophen and opioids to oral formulations. Pain Pract 2012;12(2):159-73.

2. Wininger SJ, Miller H, Minkowitz HS, Royal MA, Ang RY, Breitmeyer JB, Singla NK. A randomized, double-blind, placebo-controlled, multicenter, repeat-dose study of two intravenous acetaminophen dosing regimens for the treatment of pain after abdominal laparoscopic surgery. Clin Ther 2010;32(14):2348-69.

3. Groudine S, Fossum S. Use of intravenous acetaminophen in the treatment of postoperative pain. J Peri Anaesth Nurs 2011;26(2):74-80. 
4. Pasero C, Stannard D. The role of intravenous acetaminophen in acute pain management: a case-illustrated review. Pain Manag Nurs 2012;13(2):107-24.

5. Zafar N, Davies R, Greenslade GL, Dixon AR. The evolution of analgesia in an 'accelerated' recovery programme for resectional laparoscopic colorectal surgery with anastomosis. Colorectal Dis 2010;12(2):119-24.

6. Rana S, Verma RK, Singh J, Chaudhary SK, Chandel A. Magnesium Sulphate as an adjuvant to bupivacaine in ultrasound-guided transversus abdominis plane block in patients scheduled for total abdominal hysterectomy under subarachnoid block. Indian J Anaesth 2016;60(3):174.

7. Johns N, O'neill S, Ventham NT, Barron F, Brady RR, Daniel T. Clinical effectiveness of transversus abdominis plane (TAP) block in abdominal surgery: a systematic review and metaanalysis. Colorectal Dis 2012;14(10):e635-42.

8. De Oliveira GS, Castro-Alves LJ, Nader A, Kendall MC, McCarthy RJ. Transversus abdominis plane block to ameliorate postoperative pain outcomes after laparoscopic surgery: a meta-analysis of randomized controlled trials. Anaesth Analgesia 2014;118(2):454-63.

9. Favuzza J, Delaney CP. Outcomes of discharge after elective laparoscopic colorectal surgery with transversus abdominis plane blocks and enhanced recovery pathway. J Am Coll Surg 2013;217(3):503-6.

10. Akkaya A, Yildiz I, Tekelioglu UY, Demirhan A, Bayir H, Ozlu T, Bilgi M, Kocoglu H. Dexamethasone added to levobupivacaine in ultrasound-guided tranversus abdominis plain block increased the duration of postoperative analgesia after caesarean section: a randomized, double blind, controlled trial. Eur Rev Med Pharmacol Sci 2014;18(5):717-22.

11. Simpson D, Curran MP, Oldfield V, Keating GM. Ropivacaine. Drugs 2005;65(18):2675-717.

12. Jalil RM, Yahya N, Sulaiman O, Mat WR, Teo R, Izaham A, Rahman RA. Comparing the effectiveness of ropivacaine $0.5 \%$ versus ropivacaine $0.2 \%$ for transabdominis plane block in providing postoperative analgesia after appendectomy. Acta Anaesthesiologica Taiwanica 2014;52(2):49-53.

13. Griffiths JD, Le NV, Grant S, Bjorksten A, Hebbard P, Royse C. Symptomatic local anaesthetic toxicity and plasma ropivacaine concentrations after transversus abdominis plane block for Caesarean section. Br J Anaesth 2013;110(6):9961000 .

14. Kadam VR, Moran JL. Epidural infusions versus transversus abdominis plane (TAP) block infusions: retrospective study. $J$ Anaesth 2011;25(5):786-7.

15. Singh R, Kumar N, Jain A, Joy S. Addition of clonidine to bupivacaine in transversus abdominis plane block prolongs postoperative analgesia after cesarean section. J Anaesthesiol, Clin Pharmacol 2016;32(4):501.

16. Almarakbi WA, Kaki AM. Addition of dexmedetomidine to bupivacaine in transversus abdominis plane block potentiates post-operative pain relief among abdominal hysterectomy patients: A prospective randomized controlled trial. Saudi $J$ Anaesth 2014;8(2):161.

17. Metwally AA, Abo-El-Enin KM, Allah SI, Soliman NM, AboOmar WA. Ultrasound-guided transversus abdominis plane block for lower abdominal surgeries: bupivacaine alone or combined with fentanyl or epinephrine. Menoufia Med J 2017;30(2):538.

18. Ra YS, Kim CH, Lee GY, Han JI. The analgesic effect of the ultrasound-guided transverse abdominis plane block after laparoscopic cholecystectomy. Korean J Anaesthesiol 2010;58(4):362-8.

19. Aveline C, Le Hetet H, Le Roux A, Vautier P, Cognet F, Vinet $\mathrm{E}$, Tison C, Bonnet F. Comparison between ultrasound-guided transversus abdominis plane and conventional ilioinguinal/iliohypogastric nerve blocks for day-case open inguinal hernia repair. Br J Anaesth 2010;106(3):380-6.

20. Gulhas N, Kayhan G, Sanli M, Kitlik A, Durmus M. Effect of Adding Dexamethasone to Bupivacaine on Transversus Abdominis Plane Block [Transversus Abdominis Plane Bloða Eklenen Deksametazonun Etkisi]-. Medicine Science| Int Med $J$ 2015;4(4):3732-42.

21. Chiono J, Bernard N, Bringuier S, Biboulet P, Choquet O, Morau D, Capdevila X. The ultrasound-guided transversus abdominis plane block for anterior iliac crest bone graft postoperative pain relief: a prospective descriptive study. Reg Anaesth Pain Med 2010;35(6):520-4.

22. Niraj G, Kelkar A, Hart E, Horst C, Malik D, Yeow C, Singh $\mathrm{B}$, Chaudhri S. Comparison of analgesic efficacy of fourquadrant transversus abdominis plane (TAP) block and continuous posterior TAP analgesia with epidural analgesia in patients undergoing laparoscopic colorectal surgery: an openlabel, randomised, non-inferiority trial. Anaesth 2014;69(4):348-55.

23. Almarakbi WA, Kaki AM. Addition of dexmedetomidine to bupivacaine in transversus abdominis plane block potentiates post-operative pain relief among abdominal hysterectomy patients: A prospective randomized controlled trial. Saudi $J$ Anaesth 2014;8(2):161.

24. Metwally AA, Abo-El-Enin KM, Allah SI, Soliman NM, AboOmar WA. Ultrasound-guided transversus abdominis plane block for lower abdominal surgeries: bupivacaine alone or combined with fentanyl or epinephrine. Menoufia Med J 2017;30(2):538

25. Niraj G, Kelkar A, Fox AJ. Oblique subcostal transversus abdominis plane (TAP) catheters: an alternative to epidural analgesia after upper abdominal surgery. Anaesth 2009;64(10):1137-40.

26. McDonnell JG, O'donnell B, Curley G, Heffernan A, Power C, Laffey JG. The analgesic efficacy of transversus abdominis plane block after abdominal surgery: a prospective randomized controlled trial. Anaesth Analgesia 2007;104(1):193-7.

27. Carney J, McDonnell JG, Ochana A, Bhinder R, Laffey JG. The transversus abdominis plane block provides effective postoperative analgesia in patients undergoing total abdominal hysterectomy. Anaesth Analgesia 2008;107(6):2056-60.

28. Soltani Mohammadi S, Dabir A, Shoeibi G. Efficacy of transversus abdominis plane block for acute postoperative pain relief in kidney recipients: a double-blinded clinical trial. Pain Med 2014;15(3):460-4.

29. Parikh BK, Waghmare V, Shah VR, Modi P, Rizvi S, Khemchandani S, Butala B, Parikh G. The analgesic efficacy of continuous transversus abdominis plane block in renal transplant recipients. J Anaesthesiol, Clin Pharmacol 2015;31(4):531.

How to cite this article: Shreyas S, Shivanand LK, Allalamath S, Talikoti DG. Efficacy of pre-emptive ultrasound guided transverses abdominis plane block with dexamethasone added to bupivacaine for postoperative analgesia after laparoscopic surgeries: A randomised clinical study. Indian J Clin Anaesth 2019;6(2):280-8. 